\title{
Cloning and Characterization of a Flavonol Synthase Gene from Scutellaria baicalensis
}

\author{
Yeon Bok Kim, ${ }^{1}$ KwangSoo Kim, ${ }^{2}$ YeJi Kim, ${ }^{1}$ Pham Anh Tuan, ${ }^{1}$ Haeng Hoon Kim, ${ }^{3}$ \\ Jin Woong Cho, ${ }^{1}$ and Sang Un Park ${ }^{1}$ \\ ${ }^{1}$ Department of Crop Science, Chungnam National University, 99 Daehak-ro, Yuseong-gu, Daejeon 305-764, Republic of Korea \\ ${ }^{2}$ Department of Biochemistry and Molecular Biology, Baylor College of Medicine One Baylor Plaza, Houston, TX 77030, USA \\ ${ }^{3}$ Department of Well-Being Resources, Sunchon National University, 413 Jungangno, Suncheon, Jeollanam-do 540-742, \\ Republic of Korea
}

Correspondence should be addressed to Jin Woong Cho; jwcho@cnu.ac.kr and Sang Un Park; supark@cnu.ac.kr

Received 24 August 2013; Accepted 24 October 2013; Published 28 January 2014

Academic Editors: J. Jakse and E. Porceddu

Copyright (C) 2014 Yeon Bok Kim et al. This is an open access article distributed under the Creative Commons Attribution License, which permits unrestricted use, distribution, and reproduction in any medium, provided the original work is properly cited.

\begin{abstract}
Flavonols are the most abundant of all the flavonoids and play pivotal roles in a variety of plants. We isolated a cDNA clone encoding flavonol synthase from Scutellaria baicalensis (SbFLS). The SbFLS cDNA is 1011 bp long, encodes 336 amino acid residues, and belongs to a family of 2-oxoglutarate-dependent dioxygenases. The overall structure of SbFLS is very similar to that of Arabidopsis thaliana anthocyanidin synthase (AtANS), with a $\beta$ jelly-roll fold surrounded by tens of short and long $\alpha$-helices. SbFLS was constitutively expressed in the roots, stems, leaves, and flowers, with particularly high expression in the roots and flowers. SbFLS transcript levels in the roots were 376-, 70-, and 2.5-fold higher than in the leaves, stems, and flowers. The myricetin content was significantly higher than that of kaempferol and quercetin. Therefore, we suggest that SbFLS mediates flavonol formation in the different organs of S. baicalensis. Our study may contribute to the knowledge of the role of FLS in S. baicalensis.
\end{abstract}

\section{Introduction}

Scutellaria baicalensis Georgi (Lamiaceae) is one of the most popular herbs in several oriental countries, where it is used to treat inflammation, respiratory tract infections, diarrhea, dysentery, jaundice/liver disorders, hypertension, hemorrhaging, and insomnia [1]. Scutellaria is a mild relaxant that affects the neural and muscular-skeletal systems [2]. S. baicalensis root has abundant flavones, a class of plant flavonoids [3].

Polyphenols, synthesized via the phenylpropanoid pathway, can be classified into (iso)flavonoids, coumarins, aurones, condensed tannins, and anthocyanins [4]. Flavonoids are important secondary metabolites derived from malonylCoA and the aromatic amino acid phenylalanine [5]; approximately 8000 different flavonoid compounds have been identified [6]. Flavonols are the most abundant of all the flavonoids [7] and play pivotal roles in a variety of plants [8]. Flavonols are particularly well known for their antioxidant, anti-inflammatory, antiangiogenic, antiproliferative, and neuropharmacological properties and thereby account for the health-promoting effects of grapes and many other fruits $[8,9]$. Flavonols are synthesized from dihydrokaempferol (DHK), dihydroquercetin (DHQ), or dihydromyricetin (DHM) by flavonol synthase (FLS) enzymes (Figure 1). FLS belongs to a growing family of 2-oxoglutaratedependent dioxygenase (2-ODD) nonheme ferrous enzymes that also includes the flavonoid enzymes flavanone-3 $\beta$-hydroxylase (F3H), flavone synthase I (FS I), and leucoanthocyanidin reductase (LDOX) [10]. FLS activity was first characterized in irradiated parsley cells [11]. The first FLS gene was isolated from Petunia hybrida and expressed in yeast [12]. FLS genes have recently been cloned and characterized in Populus tremula [13], Zea mays [14], Fagopyrum tataricum [15], and Ginkgo biloba [16]. Cloning and characterization of FLS from S. baicalensis have not been reported.

In this study, a full-length cDNA encoding FLS was isolated from $S$. baicalensis by using next-generation sequencing platforms (NGS) (Roche 454 FLX+ and Illumina HiSeq 2000) (unpublished data). The mRNA transcript levels and flavonol accumulation from different organs of $S$. baicalensis were 


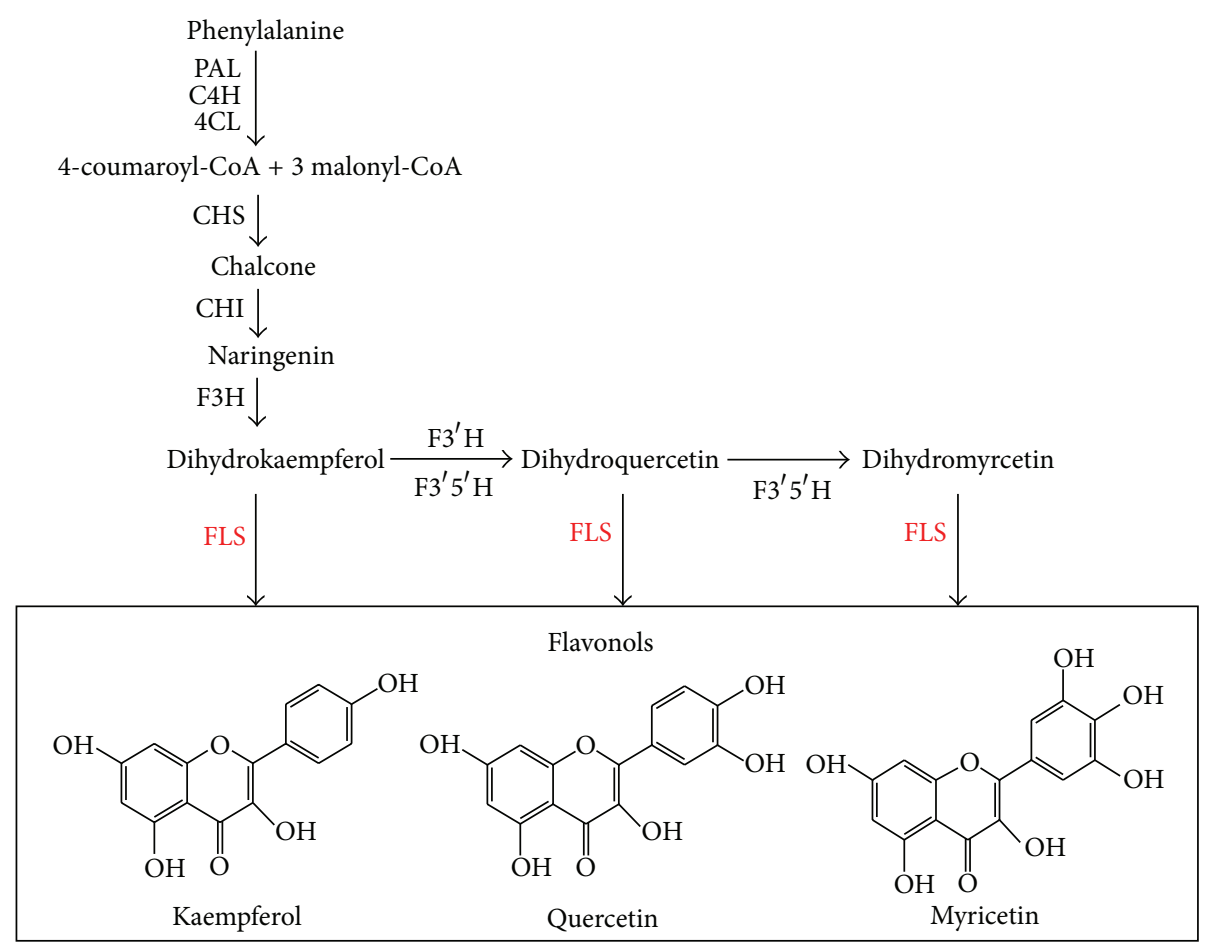

FIGURE 1: Flavonol biosynthesis in plants (redrawn from [37]). The red letter and black box indicate the enzyme and compound analyzed in this study. PAL, phenylalanine ammonia lyase; $\mathrm{C} 4 \mathrm{H}$, cinnamate 4-hydroxylase; 4CL, 4-coumaroyl CoA ligase; $\mathrm{CHS}$, chalcone synthase; $\mathrm{CHI}$, chalcone isomerase; $\mathrm{F} 3 \mathrm{H}$, flavone 3-hydroxylase; F3' $\mathrm{H}$, flavonoid $3^{\prime}$-hydroxylase; F3' $5^{\prime} \mathrm{H}$, flavonoid $3^{\prime} 5^{\prime}$-hydroxylase; FLS, flavonol synthase.

analyzed by real-time PCR and high-performance liquid chromatography (HPLC). The cloning and characterization of SbFLS may provide a foundation to elucidate the mechanism of flavonol synthesis in S. baicalensis.

\section{Materials and Methods}

2.1. Plant Material and Growth Conditions. The seeds of $S$. baicalensis were sown in May 2012, and then seedlings were transferred into pots filled with perlite-mixed soil. Seedlings were grown under $16 \mathrm{~h}$ light and $8 \mathrm{~h}$ dark conditions in the greenhouse $\left(25^{\circ} \mathrm{C}\right.$ and $50 \%$ humidity) at Chungnam National University (Daejeon, Korea). We used at least nine pots for biological repeats. Each organ (flowers, stems, leaves, and roots) was collected for two weeks after the first day of flowering. All samples were frozen in liquid nitrogen upon collection and stored at $-80^{\circ} \mathrm{C}$ prior to RNA isolation and HPLC.

2.2. Cloning of the cDNA Encoding Flavonol Synthase. A putative flavonol synthase was obtained from S. baicalensis by next-generation sequencing (NGS) (Roche $454 \mathrm{FLX}+$ and Illumina HiSeq 2000) (unpublished data). We obtained $1,226,938$ reads from Roche 454 FLX+ and 161,417,646 reads from Illumina HiSeq 2000. The de novo assembly of the 454 and Illumina high-quality reads (95\% and 75\%) were resulted more than $82 \mathrm{Mb}$ sequence length with an average contig read length of 1,614 bp in 51,188 contigs which is the long length contigs with at least $500 \mathrm{bp}$. Among these contigs, a total of 39,581 contigs were annotated by BLASTX against the public NCBI nonredundant database. In particular, putative SbFLS sequence was obtained by BLASTX for comparison. The primers for the open reading frame (ORF) were as follows: forward, 5' -ATGGAGGTTGGGAGAGTG-3'; reverse, $5^{\prime}$ TCACTGGGGAAGCTTATTAAGCTTAC- $3^{\prime}$. The TM Calculator program (http://bioinfo.ut.ee/primer3-0.4.0/) was used to compute the PCR annealing temperatures. The PCR program consisted of denaturation at $94^{\circ} \mathrm{C}$ for $1 \mathrm{~min}$, annealing at $45^{\circ} \mathrm{C}$ for $1 \mathrm{~min}$, and an extension at $72^{\circ} \mathrm{C}$ for $1 \mathrm{~min}$. Thirty cycles were preceded by denaturation step at $94^{\circ} \mathrm{C}$ for $3 \mathrm{~min}$, followed by a final extension at $72^{\circ} \mathrm{C}$ for $10 \mathrm{~min}$. The amplified product was purified and cloned into the $\mathrm{T}$ blunt vector (SolGent, Daejeon, Korea) and sequenced. The full-length FLS sequence was aligned using BioEdit Sequence Alignment Editor, version 5.0.9 [17]. For putative conserved domains, we used a GenBank conserved domain database search and function analysis service in NCBI.

2.3. Total RNA Extraction and Quantitative Real-Time RT$P C R$. Total RNA was isolated from $S$. baicalensis organs with an RNeasy Plant Mini Kit (Qiagen, Valencia, CA, USA). Firststrand cDNA was synthesized from total RNA $(1 \mu \mathrm{g})$ with ReverTra Ace- $\alpha$ - (Toyobo, Osaka, Japan) and oligo $(\mathrm{dT})_{20}$ primer according to the manufacturer's protocol. ORF and gene-specific primers were designed using an online program (http://frodo.wi.mit.edu/primers3). The gene-specific primer sets were designed for SbFLS-RT (forward), $5^{\prime}$-ACCCAACGAGGTTCAAGGAC-3', and SbFLS-RT (reverse), $5^{\prime}$-TGGTGGGGTCTCTTCATTCA-3' . Real-time PCR was performed in a $20 \mu \mathrm{L}$ reaction volume with $0.5 \mu \mathrm{M}$ each primer and $2 \times$ SYBR Green real-time PCR master mix 
(Toyobo, Osaka, Japan). Cycling conditions were as follows: 1 cycle of $95^{\circ} \mathrm{C}$ for $3 \mathrm{~min}$, followed by 40 cycles of $95^{\circ} \mathrm{C}$ for $15 \mathrm{~s}$, $72^{\circ} \mathrm{C}$ for $20 \mathrm{~s}$, and annealing at $50^{\circ} \mathrm{C}$. Real-time RT-PCR was carried out in triplicate on a CFX96 real-time PCR system (Bio-Rad; Hercules, CA, USA). The actin gene (GenBank accession number HQ847728) was used as an internal reference to standardize the cDNA template concentration.

2.4. Bioinformatic Analysis of Flavonol Synthase. The deduced amino acid sequence of SbFLS was aligned using the BioEdit Sequence Alignment Editor, version 5.0.9 (Department of Microbiology, North Carolina State University, Raleigh, NC, USA) [17]. Theoretical molecular weights (MW) and isoelectric points ( $\mathrm{pI}$ ) were calculated using the Compute pI/Mw tool (http://ca.expasy.org/tools/pi_tool.html). Putative target localization of SbFLS was predicted by using WoLF PSORT (http://psort.hgc.jp/form.html) [18]. The phylogenetic tree was constructed using an online program (http://www.phylogeny.fr/) [19]. Secondary structure prediction was investigated by network protein sequence ana-lysis at SOPMA (http://npsa-pbil.ibcp.fr/cgi-bin/npsa_automat .pl?page=/NPSA/npsa_sopma.html) [20]. The three-dimensional (3D) structure was built using the SWISS-MODEL program and illustrated with the PyMOL viewer.

2.5. HPLC Analysis. Flavonol content was determined as described in the studies by Li et al. [15] and Kovács et al. [21], with a modification. Flavonol standards, kaempferol, quercetin, and myricetin were purchased from Sigma-Aldrich. The harvested organs (flowers, stems, leaves, and roots) were freeze-dried for $48 \mathrm{~h}$ and ground into a fine powder using a mortar and pestle. Powdered samples $(100 \mathrm{mg})$ were extracted by sonication in $80 \%(\mathrm{v} / \mathrm{v})$ methanol at room temperature for $60 \mathrm{~min}$. During extraction, samples were vortexed every $15 \mathrm{~min}$. After extraction, the extracts were centrifuged at $14,000 \mathrm{rpm}$ for $10 \mathrm{~min}$ and the supernatant was filtered with a $0.45 \mu \mathrm{m}$ Acrodisc syringe filter (Pall Corp.; Port Washington, NY) for HPLC. HPLC was performed with a C18 column $(250 \times 4.6 \mathrm{~mm}, 5 \mu \mathrm{m}$; RStech; Daejeon, Korea $)$. The mobile phase was a gradient prepared from mixtures of methanol and $0.1 \%$ acetic acid. The flow rate was maintained at $0.7 \mathrm{~mL} / \mathrm{min}$. An injection volume of $20 \mu \mathrm{L}$ and wavelength of $270 \mathrm{~nm}$ were used for detection. The compounds in the sample were determined using a standard curve. Determinations were performed after 3 separate extractions of each sample and each extract was analyzed in triplicate $(n=3)$.

\section{Results and Discussion}

3.1. Cloning and Sequencing Analysis of FLS. Based on S. baicalensis NGS data (unpublished), we designed primers specific to the open reading frame (ORF) and flower cDNA to generate a $1011 \mathrm{bp}$ full-length fragment for use in PCR. The SbFLS amino acid sequence obtained from the PCR fragment contained 1 more residue than indicated by the NGS data (data not shown). SbFLS (GenBank accession no. KC404852) was composed of 336 amino acids with a MW and pI of $38.3 \mathrm{kDa}$ and 5.51, respectively. This result corresponded

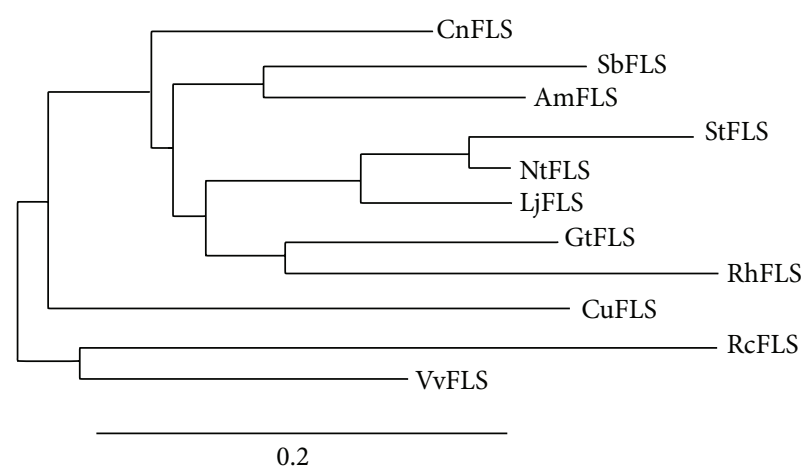

Figure 2: Phylogenetic relationships of FLS protein from $S$. baicalensis and selected species. GenBank accession numbers are as follows: NtFLS (Nicotiana tabacum, ABE28017), AmFLS (Antirrhinum majus, ABB53382), CnFLS (Camellia nitidissima, ADZ28516), StFLS (Solanum tuberosum, ACN81826), GtFLS (Gentiana triflora, BAK09226), VvFLS (Vitis vinifera, BAE75809), RhFLS (Rudbeckia hirta, ABN79672), CuFLS (Citrus unshiu, BAA36554), LjFLS (Lonicera japonica, AFJ44313), RcFLS (Ricinus communis, XP_002513772), and SbFLS (S. baicalensis, KC404852).

with the published sequence from Arabidopsis [22]. G. biloba FLS has a 1023 bp ORF encoding a 340 amino acid protein, MW of $38.7 \mathrm{kDa}$, and pI of 5.75 [16]. The deduced SbFLS shared $77,74,72,72,71$, and $70 \%$ identity with FLS proteins from Antirrhinum majus (ABB53382), Nicotiana tabacum (ABE28017), Camellia nitidissima (ADZ28516), Gentiana triflora (BAK09226), Solanum tuberosum (ACN81826), and Vitis vinifera (BAE75809), respectively (Supplementary Figure S1 available online at http://dx.doi.org/10.1155/2014/ 980740). Owens et al. [23] reported that the genome of Arabidopsis thaliana contains 5 sequences with high similarity to AtFLS1, a previously characterized flavonol synthase gene; 4 AtFLS isoforms are located on chromosome 5. A GenBank conserved domain database search and function analysis revealed that SbFLS has putative conserved domains belonging to the 20G-FeII_Oxy super family and the highly conserved $\mathrm{N}$-terminal region of proteins with 2-oxoglutarate/ $\mathrm{Fe}(\mathrm{II})$-dependent dioxygenase activity. This is consistent with the results reported by $\mathrm{Xu}$ et al. [16]. Moreover, SbFLS had conserved sequence motifs, including the HXD motif [15] for ligating ferrous iron and the RXS motif for binding 2oxoglutarate (2OG) [16]. To determine the relationship between the putative SbFLS protein and other plant FLSs, we performed phylogenetic analysis (Figure 2). The SbFLS phylogeny was clustered into 2 distinct groups and showed the closest relationship with $A$. majus. The subcellular targeting of SbFLS was predicted by PSORT to be nuclear and cytosolic. AtFLS1 localizes to the cytoplasm and nucleus [22], whereas maize FLS localizes in the ER and perinuclear region [24]. SOPMA [20] indicated that SbFLS contains 121 (36\%) alphahelices, $61(18.6 \%)$ extended strands, 22 (6.6\%) beta turns, and $132(39.3 \%)$ random coils, respectively.

3.2. Modeled 3D-Structure of SbFLS. To identify the catalytic residues, SbFLS was homologously modeled on the $1.75 \AA$ resolution structure of $A$. thaliana anthocyanidin 


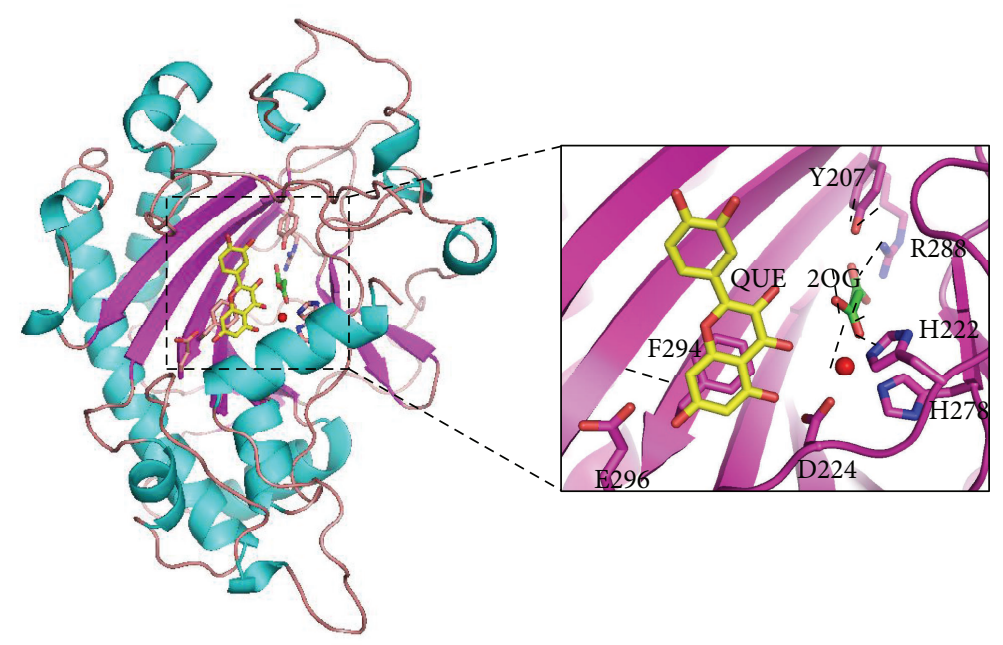

(a)

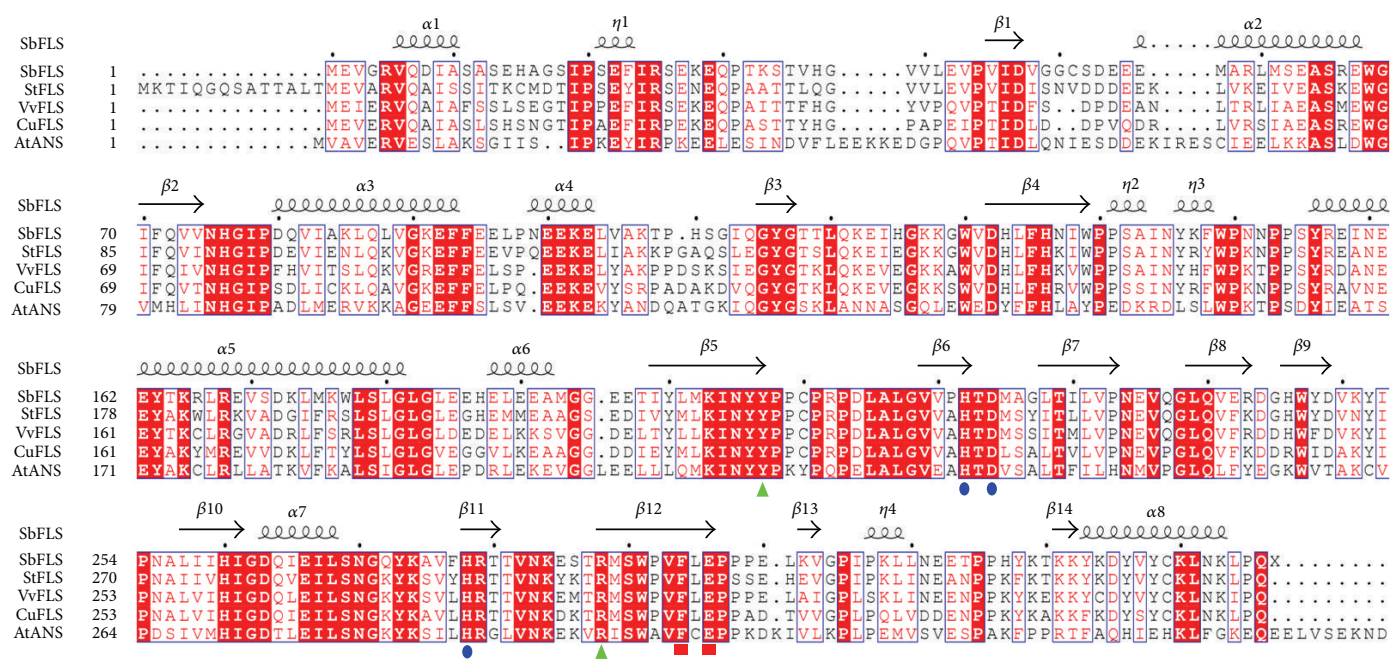

(b)

Figure 3: The structural model of SbFLS. (a) The overall and detailed views of the structure of SbFLS showing the $\beta$ jelly-roll fold and presumed catalytic site are modeled using SWISS-MODEL server (http://swissmodel.expasy.org/) [27]. Iron atom is represented by a red dot. Substrates quercetin (QUE) and 2-oxoglutarate (2OG) are indicated in yellow and green. The iron, QUE, and $2 \mathrm{OG}$ are positioned by the same structure identified in the AtANS complex (PDB code 1GP6). Possible hydrogen bonds are indicated by dashed lines. (b) Sequence alignment of AtANS and various FLSs from S. baicalensis (SbFLS), V. vinifera (VvFLS), C. unshiu (CuFLS), and A. thaliana (AtFLS). The secondary structure was drawn from the modeled structure of SbFLS. Residues coordinating with an iron atom are represented by blue ovals. Green triangles and red rectangles indicate residues that interact with 2OG and QUE, respectively. The figure was produced using the ESPript server (http://espript.ibcp.fr/ESPript/ESPript/) [38].

synthase (AtANS; PDB 1GP6) [25]. The overall structure of SbFLS and AtANS is very similar, with a $\beta$ jelly-roll fold surrounded by tens of short and long $\alpha$-helices (Figure 3(a)). The iron metal may be ligated with a bidentate group of cosubstrate $2 \mathrm{OG}$ as well as 3 side-chain residues, that is, $\mathrm{H} 222$, D224, and H278, which are conserved in AtANS and SbFLS (Figures 3(a) and 3(b)) $[15,25]$. Hydrogen bonds may form between the other side of $2 \mathrm{OG}$ and 3 conserved residues, that is, Y207, R288, and S290 (Figure 3(b)) [15, 25, 26]. A substrate quercetin (QUE) might be located near an iron metal ion, in which 5 invariant residues H133, F135, K203, F294, and E296 may contribute to binding stability by forming a $\pi$-stacking arrangement (especially F294) with the QUE ring and by hydrogen bonding. All interactions as shown here were less than $3.5 \AA$ distant. Kinetic analyses of AtANS revealed that most of the highly conserved residues play key roles in substrate/cosubstrate binding and enzymatic catalysis through metal coordination [27].

3.3. Transcript Levels of SbFLS in Different Tissues. Expression of SbFLS was investigated in the flowers, leaves, stems, and roots of $S$. baicalensis by qRT-PCR (Figure 4). Transcription of SbFLS was highest in the roots and lowest in the leaves. SbFLS transcription in roots was 376-, 70-, and 2.5-fold higher than in leaves, stems, and flowers. Very recently, Xu et al. [16] reported that the highest level of G. biloba FLS occurs 


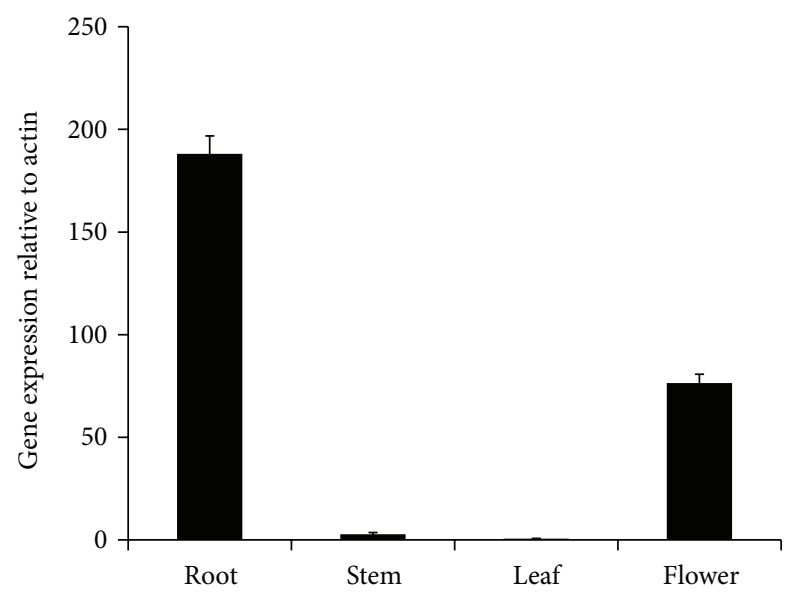

Figure 4: Transcript levels of SbFLS in different organs. The height of each bar and error bars represent means and standard errors, respectively, from 3 independent measurements.

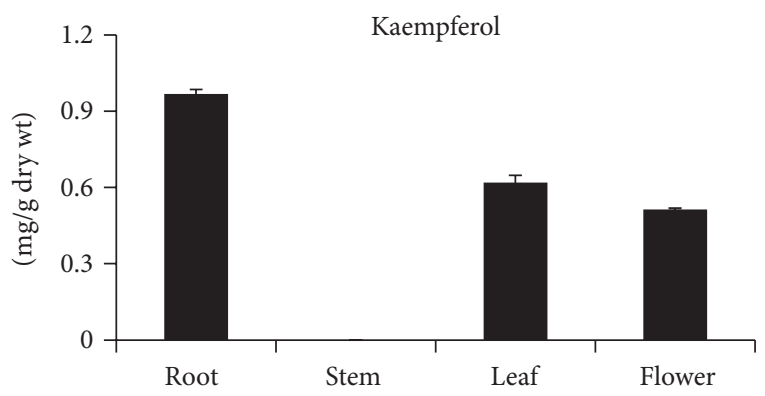

(a)

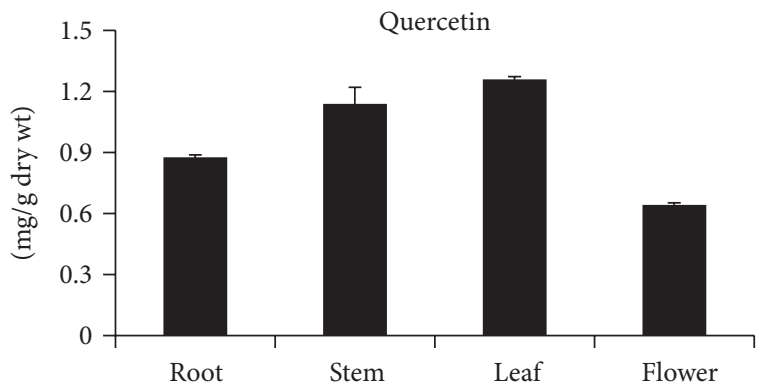

(b)

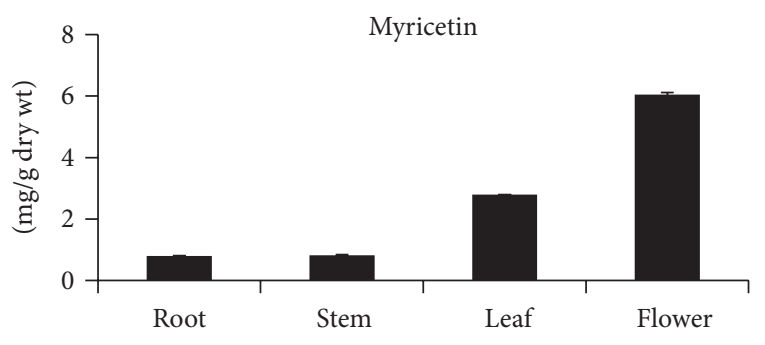

(c)

FIGURE 5: Flavonol content in different organs of S. baicalensis. The height of each bar and error bars represent the means and standard errors, respectively, from 3 independent measurements. in mature leaves, whereas the lowest level was observed in the roots. Expression of AtFLS isoforms follows different patterns in different tissues [23]. The transcription of AtFLS1 was highest in floral buds, flowers, and siliques, whereas the roots and shoots of young seedlings and the lea-ves of later vegetative stages exhibited lower expression. Other AtFLS isoforms were expressed at much lower levels or were undetectable in all tissues [23]. Therefore, Owens et al. [23] suggested that only AtFLS1 contributes to flavonol synthesis in Arabidopsis. Transcription of Citrus unshiu FLS was higher in young leaves than in old leaves and increased in the peel during fruit maturation [28]. Based on the transcription patterns in several plants, we conclude that FLS is differentially regulated in different plants and plant tissues.

3.4. Accumulation of Flavonol in Different Tissues. Kaempferol, quercetin, and myricetin were detected in almost all tissues (i.e., flowers, stems, leaves, and roots) by HPLC (Figure 5). The myricetin content was significantly higher than that of kaempferol and quercetin. The transcription pattern of SbFLS was similar to the accumulation pattern of kaempferol. Roots showed the highest kaempferol content $\left(0.97 \mathrm{mg} \mathrm{g}^{-1}\right.$ dry weight (DW)); the leaves and flowers contained similar amounts ( 0.62 and $\left.0.51 \mathrm{mg} \mathrm{g}^{-1} \mathrm{DW}\right)$, but no kaempferol was detected in the stem. Kaempferol and some glycoside derivatives have a wide range of pharmacological activities [29] and account for the utility of dry Scutellaria root as a multipurpose herb in oriental medicine. The leaves contained the highest levels of quercetin $\left(1.26 \mathrm{mg} \mathrm{g}^{-1} \mathrm{DW}\right)$, whereas the lowest amount of quercetin was in the flowers $\left(0.64 \mathrm{mg} \mathrm{g}^{-1} \mathrm{DW}\right)$. Interestingly, roots displayed the lowest amount of myricetin $\left(0.8 \mathrm{mg} \mathrm{g}^{-1} \mathrm{DW}\right)$, whereas the highest myricetin content was found in the flowers $\left(6.05 \mathrm{mg} \mathrm{g}^{-1} \mathrm{DW}\right)$. The myricetin content in the flowers was 2.2- and 7.5-fold higher than that in the leaves and roots, respectively. Feng and Liu [30] reported that the quercetin content of leaves was significantly higher than the content in pseudostems in different tissues of Welsh onion (Allium fistulosum L.).

Quercetin, kaempferol, morin, rutin, and myricetin act as antioxidants and possess anti-inflammatory, antiallergic, antiviral, and anticancer properties [31]. Quercetin is a free radical scavenger that protects against liver reperfusion ischemic tissue damage [32]. The scavenging activity of flavonols decreases as follows: myricetin $>$ quercetin $>$ kaempferol [33].

SbFLS transcription was the highest in the roots, correlating with kaempferol content in this organ. Thus, SbFLS might regulate the biosynthesis of kaempferol in S. baicalensis. Unlike kaempferol, quercetin and myricetin were most highly concentrated in the leaves and flowers, respectively. The expression level of SbFLS was not consistent with the contents of myricetin and quercetin. Owens and his colleagues [23] reported that Arabidopsis uses different isoforms of FLS with different substrate specificities to mediate the production of the quercetin and kaempferol in different tissue or cell types. In addition, Lillo et al. [34] described that FLS activity of the ANS enzyme may contribute to the differential accumulation of kaempferol and quercetin. Moreover, it was pointed out that differential expression of the $\mathrm{F} 3^{\prime} \mathrm{H}$ enzyme could also 
mediate kaempferol and quercetin ratio in petunia flowers [35]. Stracke et al. [36] reported that FLS1 could be activated by flavonol-specific transcription factors (TFs) MYB11, MYB12, and MYB111 of A. thaliana and these TFs caused different spatial accumulation of specific flavonol derivatives in leaves, stems, inflorescences, siliques, and roots [23]. Several isoforms of FLS have been isolated in other plants such as Arabidopsis [23] and maize [24]. Therefore, we presume that SbFLS isoforms, $\mathrm{SbANS}$, and $\mathrm{SbF}^{\prime} \mathrm{H}$ may be contributed to the biosynthesis of quercetin and myricetin.

\section{Conclusion}

We have described the molecular cloning and characterization of a S. baicalensis gene encoding SbFLS. Our results suggest that SbFLS is a key potential target for the flux of the flavonol biosynthesis pathway in S. baicalensis. To explain adequately the flavonol biosynthesis mechanisms in $S$. baicalensis, in vitro enzyme assay of SbFLS isoforms, SbANS, and $\mathrm{SbF}^{\prime} \mathrm{H}$ should be examined in the near future. Our study may help to determine the role of FLS and facilitate metabolic engineering of flavonol biosynthesis in S. baicalensis.

\section{Abbreviations \\ HPCL: High-performance liquid chromatography \\ CGA: Chlorogenic acid \\ PAL: Phenylalanine ammonia-lyase \\ C4H: Cinnamic acid 4-hydroxylase \\ 4CL: 4-coumaroyl:CoA-ligase \\ CHI: Chalcone isomerase \\ F3H: Flavone 3-hydroxylase \\ F3' $\mathrm{H}$ : Flavonoid $3^{\prime}$-hydroxylase \\ $\mathrm{F}^{\prime} 5^{\prime} \mathrm{H}$ : Flavonoid $3^{\prime} 5^{\prime}$-hydroxylase \\ FLS: Flavonol synthase \\ ANS: Anthocyanidin synthase.}

\section{Conflict of Interests}

The authors declare that there is no conflict of interests regarding the publication of this paper.

\section{Acknowledgment}

This work (K13101) was supported by the Korea Institute of Oriental Medicine (KIOM) Grant funded by the Korean government.

\section{References}

[1] M. Li-Weber, "New therapeutic aspects of flavones: the anticancer properties of Scutellaria and its main active constituents Wogonin, Baicalein and Baicalin," Cancer Treatment Reviews, vol. 35, no. 1, pp. 57-68, 2009.

[2] D. Y. Zhang, J. Wu, F. Ye et al., "Inhibition of cancer cell proliferation and prostaglandin E2 synthesis by Scutellaria baicalensis," Cancer Research, vol. 63, no. 14, pp. 4037-4043, 2003.

[3] S. Martens and A. Mithöfer, "Flavones and flavone synthases," Phytochemistry, vol. 66, no. 20, pp. 2399-2407, 2005.
[4] B. Winkel-Shirley, "Flavonoid biosynthesis. A colorful model for genetics, biochemistry, cell biology, and biotechnology," Plant Physiology, vol. 126, no. 2, pp. 485-493, 2001.

[5] B. W. Shirley, W. L. Kubasek, G. Storz et al., "Analysis of Arabidopsis mutants deficient in flavonoid biosynthesis," Plant Journal, vol. 8, no. 5, pp. 659-671, 1995.

[6] F. Ververidis, E. Trantas, C. Douglas, G. Vollmer, G. Kretzschmar, and N. Panopoulos, "Biotechnology of flavonoids and other phenylpropanoid-derived natural products. Part I: chemical diversity, impacts on plant biology and human health," Biotechnology Journal, vol. 2, no. 10, pp. 1214-1234, 2007.

[7] H. A. Stafford, Flavonoid Metabolism, CRC Press, Boca Raton, Fla, USA, 1990.

[8] B. H. Havsteen, "The biochemistry and medical significance of the flavonoids," Pharmacology and Therapeutics, vol. 96, no. 2-3, pp. 67-202, 2002.

[9] E. Butelli, L. Titta, M. Giorgio et al., "Enrichment of tomato fruit with health-promoting anthocyanins by expression of select transcription factors," Nature Biotechnology, vol. 26, no. 11, pp. 1301-1308, 2008.

[10] L. Britsch, J. Dedio, H. Saedler, and G. Forkmann, "Molecular characterization of flavanone $3 \beta$-hydroxylases. Consensus sequence, comparison with related enzymes and the role of conserved histidine residues," European Journal of Biochemistry, vol. 217, no. 2, pp. 745-754, 1993.

[11] L. Britsch, W. Heller, and H. Grisebach, "Conversion of flavanone to flavones, dihydroflavonol, and flavonol with an enzyme system from cell cultures of parsley," Zeitschrift Für Naturforschung, vol. 36, pp. 742-750, 1981.

[12] T. A. Holton, F. Brugliera, and Y. Tanaka, "Cloning and expression of flavonol synthase from Petunia hybrida," Plant Journal, vol. 4, no. 6, pp. 1003-1010, 1993.

[13] B.-G. Kim, E. J. Joe, and J.-H. Ahn, "Molecular characterization of flavonol synthase from poplar and its application to the synthesis of 3-O-methylkaempferol," Biotechnology Letters, vol. 32, no. 4, pp. 579-584, 2010.

[14] M. L. F. Ferreyra, S. Rius, J. Emiliani et al., "Cloning and characterization of a UV-B-inducible maize flavonol synthase," Plant Journal, vol. 62, no. 1, pp. 77-91, 2010.

[15] C. Li, Y. Bai, S. Li et al., "Cloning, characterization, and activity analysis of a flavonol synthase gene FtFLS1 and its association with flavonoid content in tartary buckwheat," Journal of Agricultural and Food Chemistry, vol. 60, pp. 5161-5168, 2012.

[16] F. Xu, L. Li, W. Zhang et al., "Isolation, characterization, and function analysis of a flavonol synthase gene from Ginkgo biloba," Molecular Biology Reports, vol. 39, no. 3, pp. 2285-2296, 2012.

[17] T. A. Hall, "BioEdit: a user-friendly biological sequence alignment editor and analysis program for Windows 95/98/NT," Nucleic Acids Symposium Series, vol. 41, pp. 95-98, 1999.

[18] P. Horton, K.-J. Park, T. Obayashi et al., "WoLF PSORT: protein localization predictor," Nucleic Acids Research, vol. 35, pp. W585-W587, 2007.

[19] A. Dereeper, V. Guignon, G. Blanc et al., "Phylogeny.fr: robust phylogenetic analysis for the non-specialist," Nucleic Acids Research, vol. 36, pp. W465-W469, 2008.

[20] C. Geourjon and G. Deleage, "SOPMA: significant improvements in protein secondary structure prediction by consensus prediction from multiple alignments," Computer Applications in the Biosciences, vol. 11, no. 6, pp. 681-684, 1995. 
[21] G. Kovács, I. N. Kuzovkina, É. Szoke, and L. Kursinszki, "HPLC determination of flavonoids in hairy-root cultures of Scutellaria baicalensis Georgi," Chromatographia, vol. 60, pp. S81-S85, 2004.

[22] M. K. Pelletier, J. R. Murrell, and B. W. Shirley, "Characterization of flavonol synthase and leucoanthocyanidin dioxygenase genes in Arabidopsis: further evidence for differential regulation of "early" and "late" genes," Plant Physiology, vol. 113, no. 4, pp. 1437-1445, 1997.

[23] D. K. Owens, A. B. Alerding, K. C. Crosby, A. B. Bandara, J. H. Westwood, and B. S. J. Winkel, "Functional analysis of a predicted flavonol synthase gene family in Arabidopsis," Plant Physiology, vol. 147, no. 3, pp. 1046-1061, 2008.

[24] F. M. L. Falcone, M. I. Casas, J. I. Questa et al., "Evolution and expression of tandem duplicated maize flavonol synthase genes," Frontiers Plant Science, vol. 3, article 101, 2012.

[25] R. C. Wilmouth, J. J. Turnbull, R. W. D. Welford, I. J. Clifton, A. G. Prescott, and C. J. Schofield, "Structure and mechanism of anthocyanidin synthase from Arabidopsis thaliana," Structure, vol. 10, no. 1, pp. 93-103, 2002.

[26] C. S. Chua, D. Biermann, K. S. Goo, and T.-S. Sim, "Elucidation of active site residues of Arabidopsis thaliana flavonol synthase provides a molecular platform for engineering flavonols," Phytochemistry, vol. 69, no. 1, pp. 66-75, 2008.

[27] K. Arnold, L. Bordoli, J. Kopp, and T. Schwede, "The SWISSMODEL workspace: a web-based environment for protein structure homology modelling," Bioinformatics, vol. 22, no. 2, pp. 195-201, 2006.

[28] T. Moriguch, M. Kita, K. Ogawa, Y. Tomono, T. Endo, and M. Omura, "Flavonol synthase gene expression during citrus fruit development," Physiologia Plantarum, vol. 114, no. 2, pp. 251258, 2002.

[29] J. M. Calderón-Montaño, E. Burgos-Morón, C. Pérez-Guerrero, and M. López-Lázaro, "A review on the dietary flavonoid kaempferol," Mini-Reviews in Medicinal Chemistry, vol. 11, no. 4, pp. 298-344, 2011.

[30] X. Feng and W. Liu, "Variation of quercetin content in different tissues of welsh onion (Allium fistulosum L.)," African Journal of Agricultural Research, vol. 6, no. 26, pp. 5675-5679, 2011.

[31] B. Halliwell, "Free radicals, antioxidants, and human disease: curiosity, cause, or consequence?" The Lancet, vol. 344, no. 8924, pp. 721-724, 1994.

[32] C. G. Fraga, V. S. Martino, and G. E. Ferraro, "Flavonoids as antioxidants evaluated by in vitro and in situ liver chemiluminescence," Biochemical Pharmacology, vol. 36, no. 5, pp. 717-720, 1987.

[33] A. K. Ratty and N. P. Das, "Effects of flavonoids on nonenzymatic lipid peroxidation: structure-activity relationship," Biochemical Medicine and Metabolic Biology, vol. 39, no. 1, pp. 6979, 1988.

[34] C. Lillo, U. S. Lea, and P. Ruoff, "Nutrient depletion as a key factor for manipulating gene expression and product formation in different branches of the flavonoid pathway," Plant, Cell and Environment, vol. 31, no. 5, pp. 587-601, 2008.

[35] D. Lewis, M. Bradley, S. Bloor et al., "Altering expression of the flavonoid 3/-hydroxylase gene modified flavonol ratios and pollen germination in transgenic Mitchell petunia plants," Functional Plant Biology, vol. 33, no. 12, pp. 1141-1152, 2006.

[36] R. Stracke, H. Ishihara, G. Huep et al., "Differential regulation of closely related R2R3-MYB transcription factors controls flavonol accumulation in different parts of the Arabidopsis thaliana seedling," Plant Journal, vol. 50, no. 4, pp. 660-677, 2007.

[37] S. Czemmel, R. Stracke, B. Weisshaar et al., "The grapevine R2R3-MYB transcription factor VvMYBF1 regulates flavonol synthesis in developing grape berries," Plant Physiology, vol. 151, no. 3, pp. 1513-1530, 2009.

[38] P. Gouet, E. Courcelle, D. I. Stuart, and F. Métoz, "ESPript: analysis of multiple sequence alignments in PostScript," Bioinformatics, vol. 15, no. 4, pp. 305-308, 1999. 

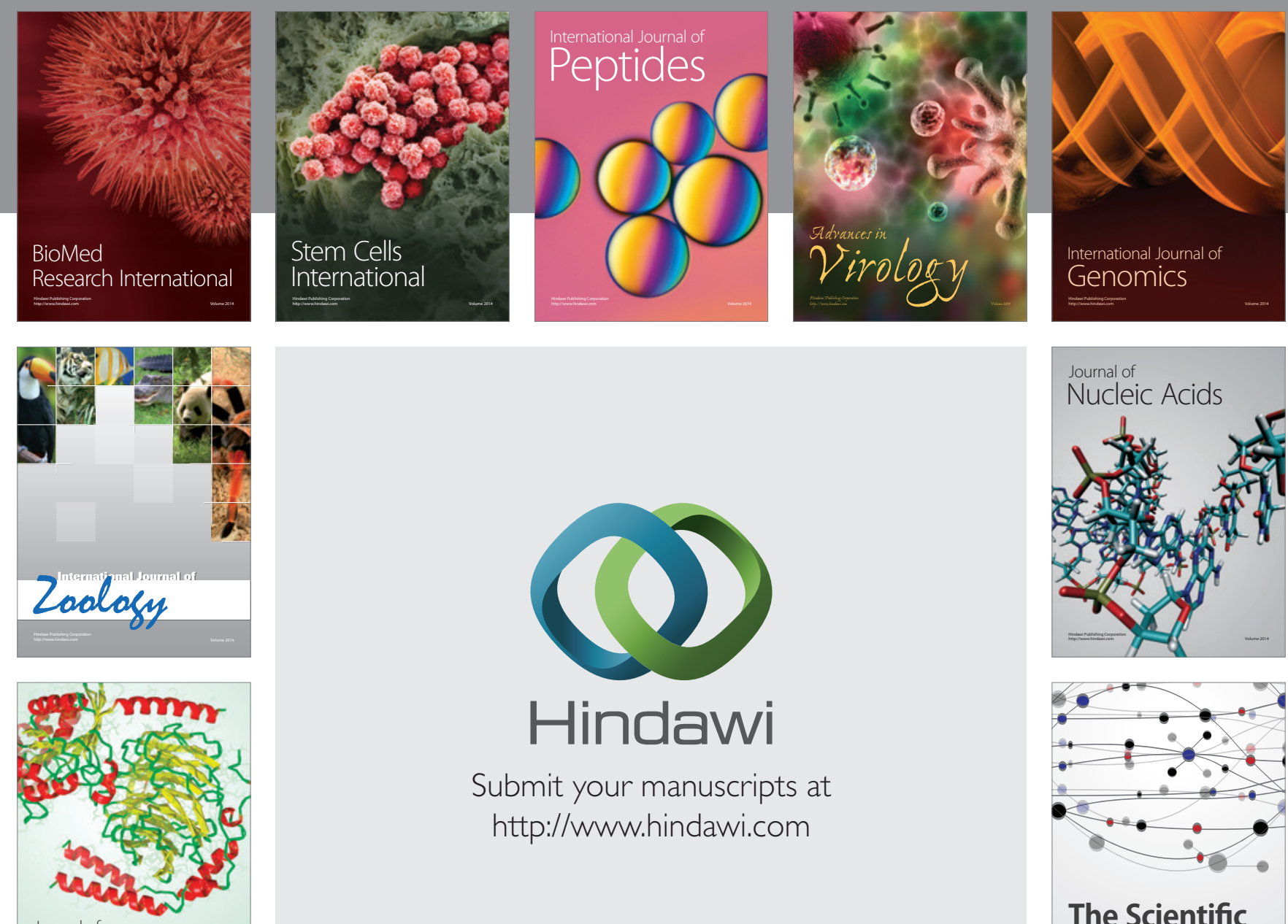

Submit your manuscripts at

http://www.hindawi.com

Journal of
Signal Transduction
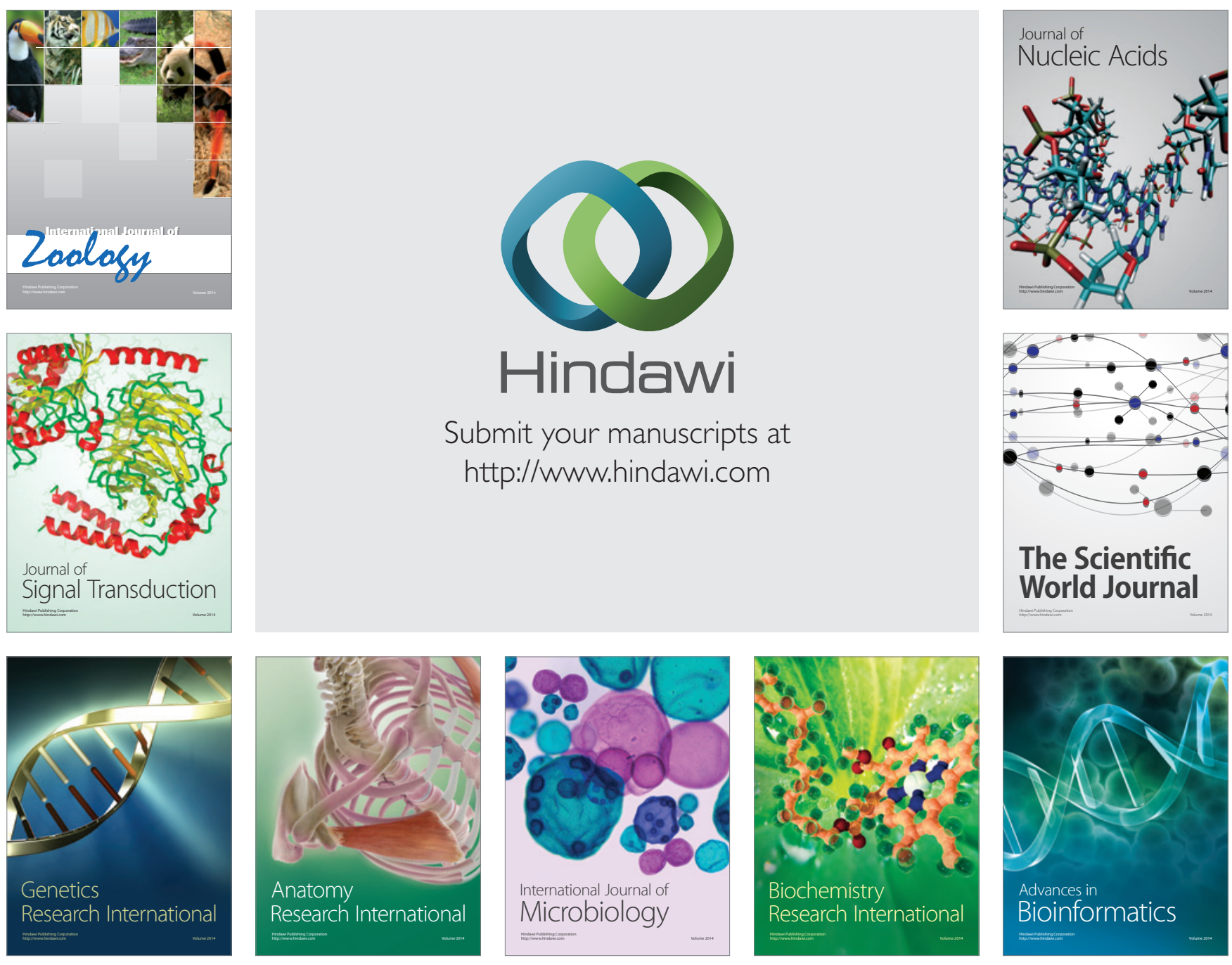

The Scientific World Journal
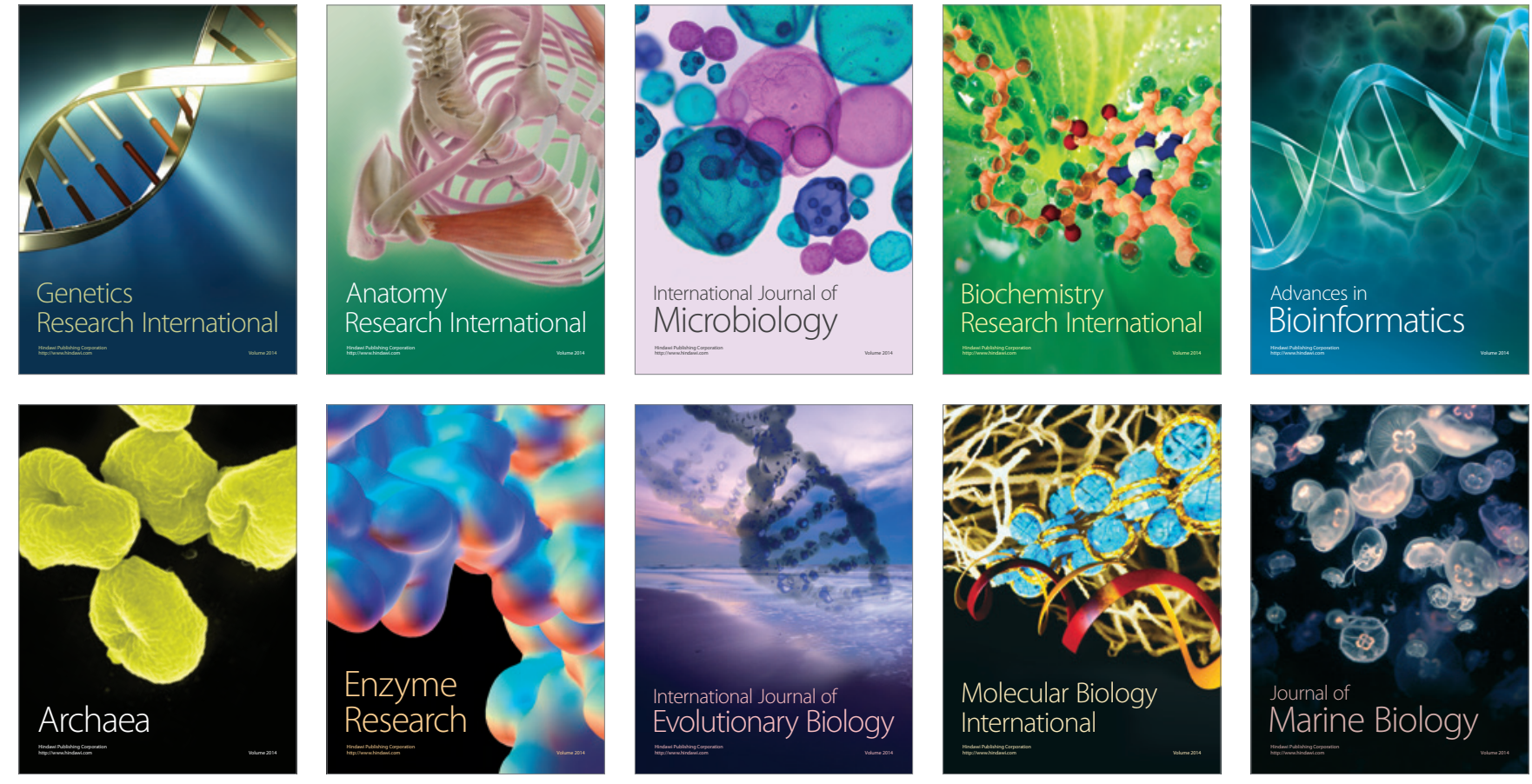